\title{
IDENTIFICATION OF DUAL mTORC1 AND mTORC2 INHIBITORS IN MELANOMA CELLS: PRODIGIOSIN vs. OBATOCLAX
}

${ }^{1}$ Espona-Fiedler M, ${ }^{1}$ Soto-Cerrato V, ${ }^{2}$ Hosseini A, ${ }^{3}$ Lizcano JM, ${ }^{2}$ Guallar V, ${ }^{4}$ Quesada R, ${ }^{5}$ Gao T and ${ }^{1}$ Pérez-Tomás R.

${ }^{1}$ Cancer Cell Biology Research Group, Department of Pathology and Experimental Therapeutics. Faculty of Medicine. University of Barcelona. E-08907 Barcelona, Spain. ${ }^{2}$ ICREA Joint BSC-IRB Research Program in Computational Biology. Barcelona Supercomputing Center. E-08034 Barcelona, Spain

${ }^{3}$ Institute of Neuroscience and Department of Biochemistry and Molecular Biology - Faculty of Medicine, Autonomous University of Barcelona. E-08193 Barcelona, Spain. ${ }^{4}$ Departament of Chemistry, Faculty of Science, University of Burgos. E-09001 Burgos, Spain.

${ }^{5}$ Markey Cancer Center, Department of Molecular and Cellular Biochemistry, University of Kentucky, Lexington, Kentucky 40536-0509.

Running title: New dual mTORC1 and mTORC2 inhibitors

Key words: prodigiosin, obatoclax, melanoma, PI3K/AKT, mTOR inhibitors, mTOR complexes

Abbreviation list: PG, prodigiosin; OBX, obatoclax; Rap, rapamycin; mTOR, mammalian target of rapamycin; PI3K, phosphoinositide 3-kinase; AKT/PKB, protein kinase B. 
Corresponding author: Ricardo Pérez-Tomás, Department of Pathology and Experimental Therapeutics, Cancer Cell Biology Research Group, University of Barcelona. Pavelló Central, 5a planta, LR 5101 C/Feixa Llarga s/n, E 08907 L'Hospitalet de Llobregat, Barcelona, Spain. Phone/Fax: +34 934024288;

E-mail: rperez@ub.edu

\section{Abstract}

The PI3K/AKT/mTOR signaling pathway regulates cell proliferation, survival and angiogenesis. The mammalian target of rapamycin (mTOR) is a protein kinase ubiquitously expressed within cells that regulates cell growth and survival by integrating nutrient and hormonal signals. mTOR exists in two complexes, mTORC1 and mTORC2. Hyperactivation of the mTOR protein has been linked to development of cancer, raising mTOR as an attractive target for cancer therapy. Prodigiosin (PG) and Obatoclax (OBX), two members of the prodiginines family, are small molecules with anticancer properties which are currently under clinical trials. In the present paper, we demonstrate that mTOR is a molecular target of both prodiginines in melanoma, a highly drug-resistant cancer model. The inhibition of mTORC1 and mTORC2 complexes by PG or OBX resulted in a loss of AKT phosphorylation at S473, preventing its full activation, with no significant effect on T308. The strongest activity inhibition (89\%) was induced by PG on mTORC2. Binding assays using Surface Plasmon Resonance (SPR) provide kinetic and affinity data of the interaction of these small molecules with mTOR. In addition, in silico modelling produced a detailed atomic description of the binding modes. These results provide new data to understand the mechanism of action of these molecules, and provide new structural data that will allow the development of more specific mTOR inhibitors for cancer treatment. 


\section{Introduction}

Prodigiosin (PG) and Obatoclax (OBX) are two prodiginine family members which have emerged as promising anticancer drugs and are currently in clinical trials. Prodiginines are bacterial metabolites with a pyrrolylpyrromethene skeleton which have shown immunosuppressive and anticancer properties. PG has shown apoptotic activity against several cancer cell types with low cytotoxicity in non-malignant cells. The National Cancer Institute (dtp.nci.nih.gov) tested prodigiosin (and some of its derivates) against a collection of $\sim 60$ cell lines with an average $\mathrm{IC}_{50}$ (for $\mathrm{PG}$ ) of $2.1 \mu \mathrm{M}$ (1). It has been described that the apoptotic process triggered by PG is mediated through the mitochondrial pathway and involves the induction of the proapoptotic gene NAG-1 (2). Nevertheless, the molecular target of this agent is still unknown. Further studies are also necessary to understand the mechanism of action of OBX. OBX is a synthetic indolylprodigiosin derivative, which was developed by GeminX Pharmaceuticals (recently acquired by Cephalon) and was described as a BH3 mimetic drug (3).

In the present report we identify the mammalian target of rapamycin (mTOR) as a new molecular target of the prodiginines. mTOR is an evolutionarily conserved serine/threonine protein kinase which is constituted by two signaling complexes: mTOR complex 1 (mTORC1) and mTOR complex 2 (mTORC2). Both complexes have specific effects on distinct cellular functions, such as controlling mRNA translation, ribosome biogenesis, autophagy and metabolism (4-6). mTORC2 phosphorylates AGC kinases such as AKT, serum- and glucocorticoid-induced protein kinase-1 (SGK1) and protein kinase C-alpha (PKC $\alpha)$ (7-9). AKT is one of the best-known downstream effectors of phosphatidylinositol-3 kinase (PI3K). Complete AKT activation depends on phosphoinositide-dependent kinase-1 
(PDK1) and mTORC2, which phosphorylate AKT at two key sites: the activation loop (T308) and the C-terminal hydrophobic motif (S473), respectively $(10,11)$. mTOR signaling is regulated through a network of feedback loops, protein partners, substrates, and regulators $(12,13)$. Among them, PRAS40 (proline-rich AKT substrate $40 \mathrm{kDa}$ ) is a key regulator of mTORC1. Moreover, in contrast to mTORC2, which contains rictor (rapamycin-insensitive companion of mTOR), mTORC1 contains raptor (regulatory associated protein of mTOR), which positively regulates mTOR activity and functions as a scaffold for recruiting mTORC1 substrates $(14,15)$. PRAS40 interacts with raptor in insulin-deprived cells and inhibits the activation of mTORC1 pathway (16). mTORC1 regulates protein synthesis through S6-kinase and the translation repressor protein $4 \mathrm{E}-\mathrm{BP} 1$. mTORC1 phosphorylates the hydrophobic motif of p70S6K on T389 (17). On the other hand, phosphorylation of 4E-BP1 at S65 by mTORC1 prevents the binding of 4E-BP1 to the eIF4E translation initiation factor activating capdependent translation (13).

Interest in identifying and developing new mTOR inhibitors has increased since the second generation of mTOR inhibitors showed encouraging results in the treatment of different types of cancer, including melanoma (18). Melanoma is an extremely aggressive disease with high metastatic potential and notoriously strong resistance to cytotoxic agents. Development of resistance has been related to the presence of different feedback loops that link both PI3K/AKT/mTOR and mitogen activated protein kinase (MAPK) pathways. These pathways are critical to melanoma progression and both are deregulated in melanoma, but not in normal cells $(19,20)$. Thus, compounds that counteract these feedback loops are considered in cancer therapy.

Here, we report that prodiginines inhibit both mTORC1 and mTORC2 complexes and thus counteract the S6K-1/IRS-1 negative feedback loop in melanoma. Moreover, binding assays provide data on the stability and affinity of the interaction between these small 
molecules and mTOR. In addition, we describe several of the recognition motifs involved in these interactions by in silico model.

\section{Material and Methods}

\subsection{Reagents}

Prodigiosin (2-methyl-3-pentyl-6-methoxyprodigiosene) was provided by Dr. R. J. Schultz of the National Cancer Drug Synthesis and Chemistry Branch Chemotherapeutic Agents Repository (Bethesda, MD). Obatoclax, a synthetic indol-containing prodiginine, was provided by Dr. Roberto Quesada of the University of Burgos (Supplementary Fig. 1). Rapamycin (FRAP1/mTOR inhibitor) was purchased from Invitrogen (Carlsbad, CA). All stock solutions were diluted in DMSO and stored at $-20^{\circ} \mathrm{C}$.

\subsection{Cell lines and Culture Conditions}

Human melanoma cancer cell lines SK-MEL-28 and SK-MEL-5 were purchased from American Type Culture Collection (Manassas, VA).

Stable control, mTOR knockdown and raptor knockdown human colon cancer cells SW480 were generated using lentivirus-based shRNA targeting mTOR or raptor as described in (21).

All cell types were cultured in Dulbecco's Modified Eagle's Medium (DMEM, Biological Industries, Beit Haemek, Israel) supplemented with 10\% heat-inactivated foetal bovine serum (FBS; Life Technologies, Carlsbad, CA), $100 \mathrm{U} / \mathrm{ml}$ penicillin, $100 \mu \mathrm{g} / \mathrm{ml}$ 
streptomycin, and $2 \mathrm{mM}$ L- glutamine all from Biological Industries. Cells were grown at $37^{\circ} \mathrm{C}$ in a $5 \% \mathrm{CO}_{2}$ atmosphere.

\subsection{Cell Viability Assay}

Cell viability was determined by MTT assay using 3-(4, 5-dimethylthiazol-2-yl)-2,5diphenyltetrazolium bromide (Sigma-Aldrich Chemical Co., St. Louis, MO) (see Supplementary Material and Methods).

\subsection{Hoescht Staining}

Cell morphology was evaluated by fluorescence microscopy following Hoescht 33342 DNA staining (Sigma-Aldrich Chemical Co., St. Louis, MO). Cells $\left(2 \times 10^{5}\right.$ cells $\left./ \mathrm{ml}\right)$ were treated or not with PG for $24 \mathrm{~h}$. They were washed in PBS and resuspended in $2 \mu \mathrm{g} / \mathrm{ml}$ Hoescht 33342 and incubated for $30 \mathrm{~min}$ at $37^{\circ} \mathrm{C}$ in the dark. Then, cells were washed in PBS and examined under a Carl Zeiss Jena microscope.

\subsection{Kinase Profiling}

Kinase profiling was performed by The National Centre for Protein Kinase Profiling (MRC Protein Phosphorylation Unit, Dundee, UK). All kinase assays were carried out using a radioactive $\left({ }^{33} \mathrm{P}\right.$-ATP $)$ filter-binding assay in duplicate. Screening was carried out at $10 \mu \mathrm{M}$ and industry standard QC procedures were used to validate each assay.

\subsection{Immunoblot Analysis}


Cells were treated with prodiginines before insulin (Sigma-Aldrich Chemical Co., St. Louis, MO) stimulation. Adherent and floating cells were lysed in buffer (50 mM Tris $\mathrm{pH} 7.5$, $60 \mathrm{mM}$ glycerophosphate, $20 \mathrm{mM}$ sodium pyrophosphate, $2 \mathrm{mM}$ EGTA, $5 \mathrm{mM}$ EDTA, 30 $\mathrm{mM}$ NaF, $1 \mathrm{mM}$ orthovanadate, $1 \mathrm{mM}$ DTT, $1 \%$ Triton X-100, $1 \mathrm{mM}$ PMSF, $5 \mu \mathrm{M}$ pepstatin A, $10 \mu \mathrm{M}$ leupeptin). Protein concentration was determined with the BCA protein assay (Pierce, Rockford, IL) using bovine serum albumin as standard. $40 \mu \mathrm{g}$ of protein extracts was separated by SDS-PAGE and transferred to Immobilon-P membranes (Millipore, Bedford, MA). Immunoblots were developed with primary antibodies according to the manufacturer's instructions (see Supplementary Material and Methods). DMSO was used as a control.

\subsection{Immunoprecipitation and Non-radioactive Kinase Activity Assay}

mTORC1 and mTORC2 complexes were immunoprecipitated from SK-MEL5 cells (see Supplementary Material and Methods). Immunoprecipitates were assayed against recombinant protein AKT1 and p70-S6K1 (Invitrogen, Carlsbad, CA), respectively, in a final volume of $30 \mu \mathrm{l}$ containing $50 \mathrm{mM}$ Tris $\mathrm{pH} 7.5,10 \mathrm{mM}$ magnesium chloride and $10 \mu \mathrm{M}$ ATP which was incubated for $30 \mathrm{~min}$ at $30^{\circ} \mathrm{C}$ with gentle shaking. Assays were stopped by addition of $5 \mu \mathrm{L}$ Laemmli buffer and samples were then heated to $100^{\circ} \mathrm{C}$ for $5 \mathrm{~min}$. Samples were loaded on SDS-PAGE gel and analyzed by immunoblotting.

\subsection{Surface Plasmon Resonance (SPR) Assays}

SPR assays were performed using Biacore T-100 (see Supplementary Material and Methods), which is a sensitive, high-performance, flow-cell-based SPR biosensor used for the 
analysis of protein-protein or protein-small molecule interactions (22). This system incorporates software wizards which assist with the analysis of every interaction parameter, including kinetic and affinity evaluation and determination of binding specificity.

\subsection{Theoretical Methods}

Using two separate computational approaches, 1) Blast + Modeller and 2) I-TASSER, we developed a homology modelling procedure for mTOR. Both approaches identified templates from the PI3K protein kinase family with $24-28 \%$ identity, and both built similar models. Additionally, two control in silico models from the PI3K family, PDK1 (pdbcode:2PE1) and PKC-alpha (pdbcode:3IW4), were chosen for the protein-ligand simulations (see Supplementary Material and Methods).

After preparing the models, we performed a cavity search with SiteMap, which confirmed the ATP binding site as the top ranked binding cavity, followed by initial rigid ligand docking with Glide (23). For PDK1 and PKC-alpha we docked the crystallographic ligands together with PG. For mTOR we docked PG, OBX and PP242, a ligand that inhibits mTOR with an $\mathrm{IC}_{50}$ of $8 \mathrm{nM}$ (24). The induced fit was modelled by 600 iterations with PELE (Protein Energy Landscape Exploration), a stochastic method of mapping large conformational rearrangements and induced fit events in protein-ligand interactions (25). To map the change in affinity after the protein-ligand induced fit, the PELE results were clustered and representative structures were redocked with Glide.

\subsection{Statistical analysis}


For analysis of activity kinasa, results are expressed as the mean \pm S.D. of three independent experiments. Statistical analysis (ANOVA) was carried out with the Statgraphics plus 5.1. statistical package. $\mathrm{P}<0.05$ and $\mathrm{P}<0.01$ are represented by $*$ and $* *$, respectively.

\section{Results}

\subsection{Prodiginines induce cell death in melanoma cells}

To determine whether prodiginines could be potential melanoma chemotherapeutical agents, we first examined their effect on two melanoma cell lines obtained from different stages of melanoma progression according to the broadly accepted Clark model (26). We examined SK-MEL-28 (radial growth phase) cells derived from an in situ melanoma. We then examined the next progression stage SK-MEL-5 (vertical growth phase) cells derived from a metastatic site (axilary node) of a melanoma-bearing patient.

To compare the cytotoxic-inducing potential of PG and OBX on melanoma cells, we treated SK-MEL-28 and SK-MEL-5 cells with both compounds at concentrations ranging from $1 \mu \mathrm{M}$ to $8 \mu \mathrm{M}$ for 24 and $48 \mathrm{~h}$. Cell viability was reduced by PG in a dose-dependent manner. PG showed a half inhibitory concentration $\left(\mathrm{IC}_{50}\right)$ value of $4.51 \pm 0.47 \mu \mathrm{M}$ and $1.02 \pm$ $0.15 \mu \mathrm{M}$ in SK-MEL-28 and SK-MEL-5 cells, respectively (Fig. 1A and 1B). In contrast to PG, OBX had little effect on cell viability at $24 \mathrm{~h}$. At $48 \mathrm{~h}$ of treatment, OBX showed an $\mathrm{IC}_{50}$ value of $2.2 \mu \mathrm{M} \pm 0.43$ and $1.8 \mu \mathrm{M} \pm 0.21$ in SK-MEL-28 and SK-MEL-5 cells, respectively.

Previous studies showed that OBX mediates cell death through the induction of autophagy and subsequent activation of apoptosis (27). Therefore, we analyzed which cell death mechanism was triggered by PG, using caspase-3,-9 and LC-3 proteins as apoptotic and autophagic markers, respectively. After PG treatment, the cytosolic form of LC-3 (LC-3 I) 
disappeared and the form conjugated to phosphatidylethanolamine (LC-3 II) appeared, in a dose-dependent manner (Fig. 1C). It has been reported that LC-3 II is recruited to autophagosomal membranes, thus participating in autophagy (28). LC-3 II was induced at shorter time exposures than the activation of caspase-9, indicating that both processes are triggered, but that autophagy precedes apoptosis. Moreover, caspase- 3 proteolysis and the formation of apoptotic bodies corroborate the apoptotic process (Fig. 1D).

In addition, these results demonstrate that the cytotoxic effect mediated by PG is higher than by OBX in melanoma cells, especially in SK-MEL-5.

\subsection{Regulation of PI3K/AKT/mTOR and MAPK pathways is mediated by prodigiosin.}

To analyze the potential of prodiginines as protein kinase regulators a kinase profiling procedure was performed. The results showed that PG $(10 \mu \mathrm{M})$ suppressed the activity of few protein kinases from a panel of 65 protein kinases encoded by the human genome. The proteins that were significantly inhibited (> 70\%) were AKT, ribosomal S6 kinase (RSK)-2, mitogen and stress activated protein kinase (MSK)-1, serum glucocorticoid-inducible kinase (SGK)-1, calmodulin-dependent kinase (CaMK)-1 and Bruton’s tyrosine kinase (Btk) (29, 30). All these proteins participate in PI $3 \mathrm{~K} / \mathrm{AKT} / \mathrm{mTOR}$ and MAPK pathways. Interestingly, other proteins closely related to these pathways such as PDK1, insulin growth factor (IGF)-I receptor, MAPK protein kinases (extracellular related kinases (ERK)-1/2, p38, c-Jun Nterminal kinase (JNK)-1/2) and inhibitor of nuclear factor kappa-B kinase (IKK) were not inhibited. Unfortunately, other proteins of interest such as mTOR were not included in this kinase profiling (Fig. 2A). These results characterize PG as a multi-kinase inhibitor, and they provide a range of new potential targets of prodiginines, all of which play a critical role in the control of the cell cycle and tumor progression. 
To elucidate the specific molecular mechanisms that induce the cytotoxic effect of prodiginines, and based on the kinase profiling results, we studied the effect of PG and OBX on PI3K/AKT/mTOR and MAPK pathways in melanoma cells. Both pathways regulate melanoma cell death and proliferation (26).

We first examined the activation of the two key effector kinases of these pathways, AKT and ERK1/2. Phosphorylation levels of these kinases were higher in SK-MEL-28 than in SK-MEL-5 cells (Fig. 2B). These differences in the up-regulation of pro-survival protein kinases might explain why resistance to prodiginine treatment depends on the cell line.

We then analyzed the effect of PG on both pathways. Cells were treated with PG at the $\mathrm{IC}_{50}$ concentrations established as described above. PG reduced AKT phosphorylation on S473 in a time-dependent manner. Nevertheless, only a slight effect was observed on T308, and PG had no effect on PDK-1 or ERK1/2 (Fig. 2C). These results, together with the kinase profiling, indicate that mTOR signaling might be down-regulated in the presence of prodiginines.

\subsection{Inhibition of mTOR signaling by prodiginines in melanoma cells}

After observing significant inhibitory effects of PG on AKT, we sought to confirm the inhibition of the AKT/mTOR/p70S6K signaling pathway by prodiginines in melanoma cells. Thus, we examined the effect of prodiginines on the main regulators and substrates of this pathway. As shown in Figure 3A, both PG and OBX inhibited mTORC2 activity, leading to an inhibition of the insulin-stimulated phosphorylation of AKT and PRAS40. Nevertheless, it was necessary to use higher doses of $\mathrm{OBX}(10 \mu \mathrm{M})$ to obtain same effects as PG at $1 \mathrm{~h}$ of treatment. Insulin-stimulated phosphorylation of PRAS40 at T246 by AKT suppresses its mTORC1 inhibitory activity. Therefore, insulin stimulation activates mTORC1 and increases 
p70S6K phosphorylation (16). After treatment with prodiginines, p70S6K and 4E-BP1, which are directly regulated by mTORC1 were also dephosphorylated. Dephosphorylation of mTOR effectors suggests that prodiginines inhibit mTOR pathway.

To further confirm that prodiginines target mTOR pathway, we first determined whether knockdown of mTOR (sh-mTOR) or raptor (sh-raptor) in SW-480 cells prevented the cytotoxic effect induced by prodiginines. Knockdown of mTOR or raptor resulted in $47 \%$ or $74 \%$ reduction in endogenous protein expression, respectively, compared with stable control cells (sh- $\phi$ ) (Supplementary Fig. 2 A and B). The functional depletion of mTOR pathway was confirmed by decreased phosphorylation levels of p70S6K and AKT in mTOR and raptor knockdown cells (Supplementary Fig. 2C). According to protein depletion levels in both cell lines, results showed higher p70S6K inhibition in raptor knockdown cells. Complete AKT inhibition was observed after treatment with PG or OBX at $4 \mu \mathrm{M}$ for $1 \mathrm{~h}$. After $6 \mathrm{~h}$ of treatment with both prodiginines, protein expression down-regulation was induced in both mTOR effectors.

We next assessed whether depletion of mTOR activity prevented from prodiginines-mediated cell death. Stable control cells and knockdown cells were treated with a range of concentrations (0-4 $\mu \mathrm{M})$ of PG or OBX for 24 or $48 \mathrm{~h}$ (Supplementary Fig. 3A and 3B). Results showed greater cytotoxic effects in sh- $\phi$ cells after treatment with PG than OBX, as observed in melanoma cells. Nevertheless, the most significant results were obtained after 48 h of treatment. At $4 \mu \mathrm{M}$ of PG or OBX, sh-mTOR and sh-raptor cell death was reduced in a $\sim 35-40 \%$ compared with sh- $\phi$ cells (Fig. 3B), demonstrating that mTOR complexes are critical for prodiginines cytotoxic effect.

\subsection{Prodiginines counteract the activation of the S6K-1/IRS-1 negative feedback loop through mTORC1 and mTORC2 inhibition.}


We further examined the ability of PG and OBX to inhibit mTORC1 and mTORC2 complexes in melanoma cells. Inhibition of mTORC1 induces activation of S6K-1/IRS-1 negative feedback (19). Dual inhibition of mTORC1 and mTORC2 might counteract this mechanism. With this in mind, we first compared the effect of both compounds with that of rapamycin, which inhibits mTORC1, leading to an increase in AKT phosphorylation through an IGF-1R-dependent mechanism. As expected, rapamycin alone decreased phosho-p70S6K levels, while increasing phospho-AKT levels. The greatest effect of rapamycin was seen at $100 \mathrm{nM}$ (Supplementary Figure 4). As a further comparison, we co-treated both cell lines with $\mathrm{PG}\left(\mathrm{IC}_{50}\right)$ or $\mathrm{OBX}(10 \mu \mathrm{M})$ and $100 \mathrm{nM}$ rapamycin for $1 \mathrm{~h}$, before insulin stimulation. In contrast to both prodiginines alone, treatment with rapamycin induced a stronger decrease in the phospho-p70S6K levels, suggesting that mTORC1 is not completely inhibited by PG or OBX (Fig. 4A). Nevertheless, in both cell lines, PG rather than OBX counteracted the activation of this feedback loop, leaving AKT quite dephosphorylated.

To evaluate the inhibition on mTORC2 and mTORC1 complexes, further kinase activity assays were performed. We isolated active mTORC2 and mTORC1 complexes from melanoma cells after insulin stimulation. Active mTORC2 and mTORC1 were immunoprecipitated from the lysates using anti-rictor and anti-raptor antibodies, respectively. mTORC2 activity was analyzed using recombinant AKT as substrate. The inhibition of mTORC2 by PG or OBX resulted in a loss of AKT phosphorylation (Fig. 4B). We next measured the effect of both molecules on mTORC1 activity using p70S6 as a substrate (Fig. 4C). Both prodiginines markedly inhibited both mTOR complexes. The strongest inhibition (89\%) was induced by PG on mTORC2 (Fig. 4D).

\subsection{Kinetic characterization}


In order to further characterize the prodiginines as mTOR inhibitors, we first monitored the interaction between $\mathrm{mTOR}$ and prodiginines by real-time interaction analysis. We used Surface Plasmon Resonance (SPR) assays, which allow kinetic and affinity evaluation and determination of binding specificity between proteins and small molecules (22).

For binding experiments, we first immobilized the recombinant protein mTOR (aa 1360-2549) on a sensor surface. The analytes (PG and OBX) were then injected in solution over the surface. Changes in SPR response were detected even at nanomolar concentrations. The interactions of small molecule inhibitors with mTOR were analyzed, providing kinetic and affinity data in the nanomolar range (Fig. 5A and 5B). The same order of magnitude in the affinity data $\left(\mathrm{K}_{\mathrm{D}}\right)$ and the low dissociation rates $(\mathrm{Kd})$ suggest that both molecules form similar specific and stable binders.

Small molecule specificity was tested by performing interaction analysis between these compounds and another protein kinase: AKT. In this case, the data did not fit this interaction model using PG as analyte. Moreover, using OBX no high-affinity saturable component was obtained (Supplementary Fig. 5). These marked differences in the characteristics of the interactions compared to mTOR suggest that stable binding does not occur between prodiginines and AKT.

\subsection{In silico docking prediction}

To further characterize the interaction of prodiginines with mTOR, we developed a homology model of the active site sequence (residues 2131-2516). PDK1 and PKC-alpha were used as control systems. Fig. 6A shows the Glide docking scores before and after the 
PELE run modeling the induced fit procedure, together with the active site RMSD along the fit process, for all systems and ligands. As expected, we obtain good initial docking scores for the two crystallographic ligands in PDK1 and PKC-alpha, -9.2 and -12.0, respectively. Furthermore, when comparing the docked structure for each ligand with its crystal structure we obtained an RMSD of $\sim 0.3 \AA$. Such agreement, however, is expected when a ligand is docked into its crystal structure. The induced fit procedure does not significantly change the scores, and only introduces slight changes to the protein-ligand structures in the active site: 1.1 $\mathrm{A}$ and $1.3 \AA$ for LAA and LW4, respectively. Thus, the control test with both crystallographic ligands indicates good affinities and small induced fit reorganization. Next, we applied the same protocol to PG and found significantly lower initial binding scores: -6 to -7. The initial models, however, were taken from the crystal structures with bound ligands. Thus, they are biased towards the crystal ligands, requiring some induced fit in order to adapt to PG. For this purpose PELE was used (25). The induced fit procedure increased the RMSD but did not substantially improve the affinity for PG; the control experiments support the absence of inhibition in PDK1 and PKC observed in the kinase profiling. Regarding the mTOR ligand docking, the initial docking score of pp242, PG and OBX is about -7 to -8 , similar to the value measured for PG in our in silico control assays. For mTOR, however, the induced fit procedure (the same used for PDK1 and PKC-alpha) introduced significant changes. We observe a clear increase in binding affinity along with a significant active site adjustment, the RMSD increases to 2.1 and 2.3 for $\mathrm{PG}$ and $\mathrm{OBX}$, respectively. For all three ligands we obtained scores which are similar to those obtained for the crystal ligands in PDK1 and PKC-alpha. Thus, our simulation studies corroborate recent observations on pp242 (24) and also support our experimental findings with PG and OBX.

In Fig. 6B and 6C we display the detailed atomic view of the induced fit of the PG- and OBXmTOR complex, where we can observe several recognition motifs. For example, in the fit PG- 
mTOR complex, the hydrophobic environment around the pentyl side chain includes Ile2500, Ile2559 and Val2504. We also observe a stacking interaction between His2340 and the PG pyrrole rings. The most interesting feature, however, is the "H-bond ring" created by the alcohol side chain in Ser2342 and the two extreme pyrrole nitrogens. In silico mutation of this serine to a glycine reduces the glide score by 3 units, pointing to the importance of this interaction.

\section{Discussion}

Prodigiosin (PG) and obatoclax (OBX), both belong to the prodiginine family, which are pyrrole alkaloids of bacterial origin. Both molecules are promising candidates as anticancer drugs, since they have pro-apoptotic activity in a broad range of human cancer cell lines (1). Although they are currently in pre-clinical and clinical trials, respectively, further studies are necessary to identify the molecular target involved in their anticancer activity. It was hypothesized that OBX has more complex effects on melanoma cells than merely binding to and inhibiting antiapoptotic Bcl-2 family proteins (3). In the present report, we considered other survival signals to further understand the mechanism of action of prodiginines. We studied the prodiginine-mediated cytotoxic effect in different stages of melanoma progression. $\mathrm{PI} 3 \mathrm{~K} / \mathrm{mTOR}$ and MAPK signaling pathways are involved in growth and progression in melanoma. Deregulation of these pathways is associated with resistance to apoptosis, increased cell growth, cell proliferation and cell energy metabolism. It also confers to melanoma resistance to many chemotherapeutic agents (26). Prodiginines overcome this resistance, and are thus cytotoxic in both cell lines. Moreover, deregulation of these pathways might explain the higher $\mathrm{IC}_{50}$ value of SK-MEL-28 cells compared to SK-MEL-5 cells. 
Melanoma cells were more sensitive to PG than to OBX. Our results are consistent with previous reports where melanoma cells rendered more sensitive to OBX-induced apoptosis only in combination with molecules that induce reticulum stress, but not as a single agent (31). It was also described that OBX induces autophagy before apoptosis (27). Our results show that PG also activates both mechanisms. Moreover, cytotoxic effect of prodiginines was partially prevented by mTOR and raptor proteins depletion, concluding that prodigininesinduced mTOR pathway inhibition leads to cell death. We have characterized the effect of both prodiginines on critical elements of mTOR signaling such as AKT, PRAS40, p70 S6K and 4E-BP1, as well as on different compensatory mechanisms which link PI3K/mTOR and MAPK signaling $(18,20)$. Our results demonstrate that prodiginines target mTOR pathway. In contrast to rapamycin, prodiginines counteract the feedback loop triggered by mTORC1 inhibition, which leads to AKT activation through IGF-1/IRS-1 signaling (19). The effectiveness of prodiginines relies on their ability to inhibit mTOR activity. Therefore, the suppression of mTORC1 as well as mTORC2 activity implies a reduction of phosphorylation of AKT at S473.

The kinetics and affinity evaluation revealed high-affinity binding between mTOR and prodiginines. Nevertheless, although mTOR is a validated target for the treatment of cancer, it might be necessary to develop more effective prodiginine-derivates with improved chemical properties that increase their bioavailability.

The second generation of mTOR inhibitors targets the ATP site. These compounds suppress AKT phosphorylation, leading to a stronger antiproliferative effect than that of rapamycin $(18,32)$. Therefore, prodiginines effect might be closer to the second generation of mTOR inhibitors. Thus, it will be interesting to compare prodiginines with other small molecules such as AZD-8055 (AstraZeneca), INK-128 (Intellikine), OSI-027 (OSI pharmaceuticals) or pp242 which also block both mTORC1 and mTORC2 complexes. pp242 
is structurally similar to prodiginines (24). In silico models suggest that prodiginines, like pp242, could interact in the active-site of mTOR and provided several of the recognition motifs involved in their interaction. Moreover, these models also illustrate the importance of the alcohol side chain and the two extreme pyrrole nitrogens of the prodiginines for the interaction on Ser2342 of mTOR.

These findings described here by PG and OBX as mTORC1 and mTORC2 inhibitors contribute to our understanding of the molecular mechanisms of action of both molecules and provide data about their structural properties that will allow the development of moreeffective mTOR inhibitors in the future.

\section{Acknowledgements}

The authors thank Marta Taulés for technical assistance from Centres Científics i Tecnològics (Universitat de Barcelona) and Robin Rycroft for language assistance. This work was supported by a research grant from Spanish government and the European Union (FISPI10/00338).

\section{References}

1. Perez-Tomas R, Vinas M. New insights on the antitumoral properties of prodiginines. Curr Med Chem 2010;17:2222-31.

2. Soto-Cerrato V, Vinals F, Lambert JR, Kelly JA, Perez-Tomas R. Prodigiosin induces the proapoptotic gene NAG-1 via glycogen synthase kinase-3beta activity in human breast cancer cells. Mol Cancer Ther 2007;6:362-9. 
3. Nguyen M, Marcellus RC, Roulston A, Watson M, Serfass L et al. Small molecule obatoclax (GX15-070) antagonizes MCL-1 and overcomes MCL-1-mediated resistance to apoptosis. Proc. Natl. Acad. Sci. U. S. A. 2007;104:19512 -7.

4. Sarbassov DD, Ali SM, Kim DH, Guertin DA, Latek RR et al. Rictor, a novel binding partner of mTOR, defines a rapamycin-insensitive and raptor-independent pathway that regulates the cytoskeleton. Curr Bio 2004;14:1296-302.

5. Wullschleger S, Loewith R, Hall MN. TOR signaling in growth and metabolism. Cell 2006;124:471-84.

6. Guertin DA, Sabatini DM. The pharmacology of mTOR inhibition. Sci Signal 2009;2:pe24. 7. Ali SM, Sabatini DM. Structure of S6 kinase 1 determines whether raptor-mTOR or rictormTOR phosphorylates its hydrophobic motif site. J Biol Chem 2005;280:19445-8.

8. Frodin M, Antal TL, Dummler BA, Jensen CJ, Deak M et al. A phosphoserine/threoninebinding pocket in AGC kinases and PDK1 mediates activation by hydrophobic motif phosphorylation. Embo J 2002:21:5396-407.

9. Garcia-Martinez JM, Alessi DR. mTOR complex 2 (mTORC2) controls hydrophobic motif phosphorylation and activation of serum- and glucocorticoid-induced protein kinase 1 (SGK1). Biochem J 2008;416:375-85.

10. Alessi DR, Andjelkovic M, Caudwell B, Cron P, Morrice N et al. Mechanism of activation of protein kinase B by insulin and IGF-1. Embo J 1996;15:6541-51.

11. Manning BD, Cantley LC. AKT/PKB signaling: navigating downstream. Cell 2007;129:1261-74.

12. Schmelzle T, Hall MN. TOR, a central controller of cell growth. Cell 2000;103:253-62.

13. Fingar DC, Salama S, Tsou C, Harlow E, Blenis J. Mammalian cell size is controlled by mTOR and its downstream targets S6K1 and 4EBP1/eIF4E. Genes Dev 2002;16:1472-87. 
14. Hara K, Maruki Y, Long X, Yoshino K, Oshiro N et al. Raptor, a binding partner of target of rapamycin (TOR), mediates TOR action. Cell 2002;110:177-89.

15. Kim DH, Sarbassov DD, Ali SM, King JE, Latek RR et al. mTOR interacts with raptor to form a nutrient-sensitive complex that signals to the cell growth machinery. Cell 2002;110:163-75.

16. Sancak Y, Thoreen CC, Peterson TR, Lindquist RA, Kang SA et al. PRAS40 is an insulinregulated inhibitor of the mTORC1 protein kinase. Mol Cell 2007;25:903-15.

17. Jefferies HB, Fumagalli S, Dennis PB, Reinhard C, Pearson RB et al. Rapamycin suppresses 5'TOP mRNA translation through inhibition of p70s6k. Embo J 1997;16:3693704.

18. Huang S, Houghton PJ. Targeting mTOR signaling for cancer therapy. Curr Opin Pharmacol 2003;3:371-7.

19. Wan X, Harkavy B, Shen N, Grohar P, Helman LJ. Rapamycin induces feedback activation of Akt signaling through an IGF-1R-dependent mechanism. Ocogene 2007;26:1932-40.

20. Carracedo A, Ma L, Teruya-Feldstein J, Rojo F, Salmena L et al. Inhibition of mTORC1 leads to MAPK pathway activation through a PI3K-dependent feedback loop in human cancer. J Clin Invest 2008;118:3065-74.

21. Liu J, Stevens PD, Gao T. mTOR-dependent regulation of PHLPP expression controls the rapamycin sensitivity in cancer cells. J Biol Chem 2011;286(8):6510-20.

22. Nordin H, Jungnelius M, Karlsson R, Karlsson OP. Kinetic studies of small molecule interactions with protein kinases using biosensor technology. Anal Biochem 2005;340:35968.

23. Glide, version 5.6, Schrödinger, LLC, New York, NY, 2010 
24. Feldman ME, Apsel B, Uotila A, Loewith R, Knight ZA et al. Active-site inhibitors of mTOR target rapamycin-resistant outputs of mTORC1 and mTORC2. PLOS Biology 2009;7: $371-383$.

25. Borrelli K, Cossins B, Guallar V. Exploring hierarchical refinement techniques for induced fit docking with protein and ligand flexibility. J Comp Chem 2010;31:1224-35.

26. Miller AJ, Mihm MC. Melanoma. Jr N Engl J Med 2006;355:51-65.

27. Pan J, Cheng C, Verstovsek S, Chen Q, Jin Y et al. The BH3-mimetic GX15-070 induces autophagy, potentiates the cytotoxicity of carboplatin and 5-fluorouracil in esophageal carcinoma cells. Cancer Letters 2010;293:167-174.

28. Kabeya Y, Mizushima N, Yamamoto A, Oshitani-Okamoto S, Ohsumi Y et al. LC3, GABARAP and GATE16 localize to autophagosomal membrane depending on form-II formation. J Cell Sci 2004;117:2805-12.

29. Dalby KN, Morrice N, Caudwell FB, Avruch J, Cohen P. Identification of regulatory phosphorylation sites in mitogen-activated protein kinase (MAPK)-activated protein kinase1a/p90rsk that are inducible by MAPK. J Biol Chem 1998;273:1496-505.

30. Hanada M, Feng J, Hemmings BA. Structure, regulation and function of PKB/AKT--a major therapeutic target. Biochim Biophys Acta 2004;1697:3-16.

31. Jiang CC, Wroblewski D, Yang F, Hersey P, Zhang XD. Human melanoma cells under endoplasmic reticulum stress are more susceptible to apoptosis induced by the $\mathrm{BH} 3$ mimetic obatoclax. Neoplasia 2009;11(9):945-55.

32. Liu Q, Throreen C, Wang J, Sabatini D, Gray NS. mTOR mediated anti-cancer drug discovery. Drug Discov Today Ther Strateg 2009;6:47. -55.

33. Tirion MM. Large amplitude elastic motions in proteins from a single-parameter, atomic analysis. Phys Rev Lett 1996;77:1905-08. 


\section{Figures}
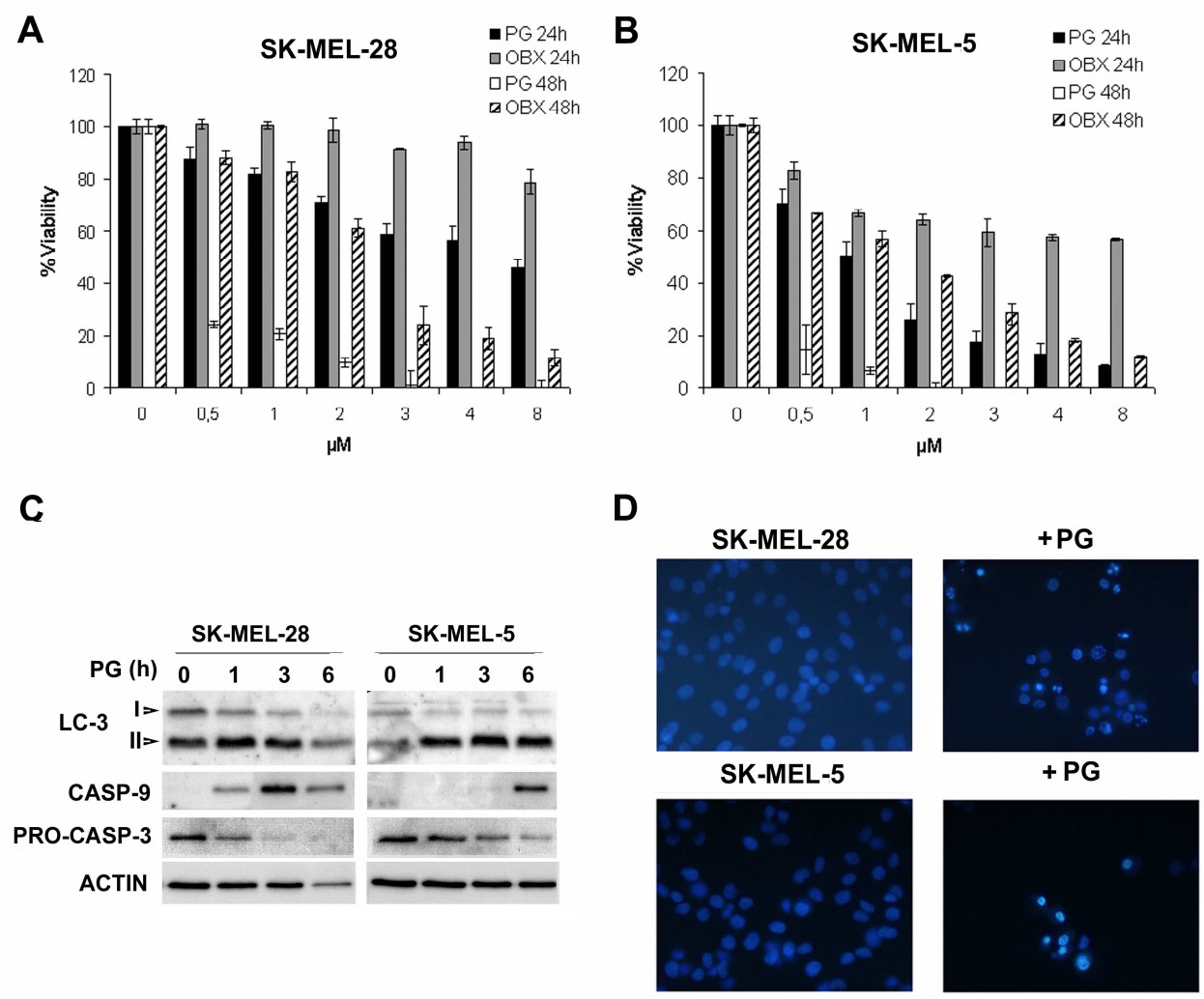

Fig. 1. Cell death in PG- or OBX-treated SK-MEL-28 and SK-MEL-5 cells. (A)

Cytotoxic effect of PG is higher than that of OBX. Cells were treated with a range of PG or OBX concentrations $(0-8 \mu \mathrm{M})$ for 24 and (B) $48 \mathrm{~h}$. Cell viability was determined by MTT assay. The percentage of viable cells was calculated as the ratio of $\mathrm{A}_{570}$ between treated and control cells. Values are shown as mean \pm S.E.M. of three independent experiments performed in triplicate. (C) PG induces activation of both autophagic and apoptotic mechanisms. Cells were treated with PG $\mathrm{IC}_{50}$ and cell extracts were assayed for LC-3, caspase-9 and procaspase-3 expression by Immunoblotting. Actin was used as loading control. (D) PG induces apoptotic body formation and nuclear condensation. This was observed through Hoescht staining in cells treated with PG $\mathrm{IC}_{50}$ for $24 \mathrm{~h}$. 
A

\begin{tabular}{|c|c|c|}
\hline Protein kinase & $\%$ activity & S.D. \\
\hline IGF1R [954-1367] & 72 & 15 \\
\hline IR [1001-1382] & 112 & 12 \\
\hline BTK [2-659] & 15 & 1 \\
\hline CaMK1 [2-369] & 19 & 5 \\
\hline MSK1 [2-802] & 32 & 3 \\
\hline RSK1 [1-735] & 69 & 10 \\
\hline RSK2 [2-740] & 22 & 1 \\
\hline SGK1 (S422D) [60-431] & 29 & 3 \\
\hline S6K1 (T412E) [1-421] & 96 & 5 \\
\hline ERK1 [2-379] & 78 & 11 \\
\hline ERK2 [2-360] & 96 & 15 \\
\hline GSK3 $\beta[2-420]$ & 48 & 19 \\
\hline p38 $\alpha$ MAPK [1-360] & 121 & 3 \\
\hline p38ßMAPK [1-364] & 100 & 19 \\
\hline p38 $\gamma$ MAPK [1-367] & 92 & 1 \\
\hline p38ठMAPK [1-365] & 97 & 1 \\
\hline IKK $\beta[1-736]$ & 66 & 12 \\
\hline JNK1 $\propto 1$ [1-384] & 77 & 16 \\
\hline JNK $2 \alpha 2[1-424]$ & 90 & 15 \\
\hline MNK1 [2-424] & 120 & 4 \\
\hline PKA [2-351] & 70 & 4 \\
\hline$P K C \alpha[1-672]$ & 98 & 3 \\
\hline PKC $\zeta$ [2-592] & 92 & 6 \\
\hline PDK1 [52-556] & 112 & 7 \\
\hline PDK1 [2-912] & 112 & 7 \\
\hline PKB $\alpha$ (S473D) [118-480] & 24 & 2 \\
\hline
\end{tabular}

B

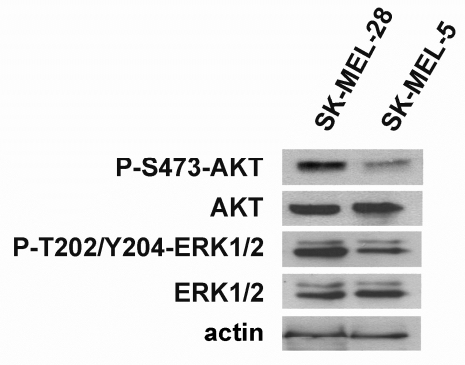

C

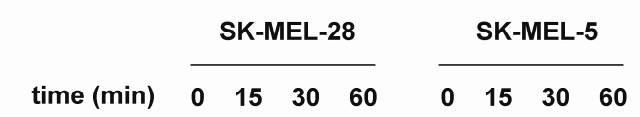

P-S473-AKT

P-T308-AKT

P-S241-PDK-1

P-T202/Y204-ERK1/2
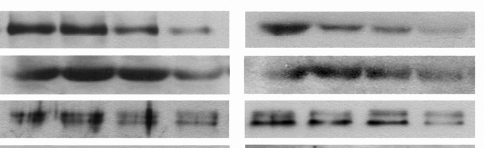

AKT

PDK-1

$=0$

ERK1/2

actin

Fig. 2. Effect of PG on PI3K/AKT/mTOR and MAPK survival pathways. (A) PG induces inhibition of some PI3K and MAPK-related protein kinases in vitro. Kinase assays were performed by the MRC Protein Phosphorylation Unit. PG $10 \mu \mathrm{M}$ was screened in duplicate against a panel of 65 protein kinases. All assays were carried out using a radioactive $\left({ }^{33} \mathrm{P}\right.$-ATP) filter-binding assay. (B) PI3K/AKT and MAPK pathways are activated in SKMEL28 more strongly than in SK-MEL-5 melanoma cells. Phosphorylation and total protein levels of AKT and ERK1/2 were visualized by Immunoblotting. Actin was used as a loading control. (C) PG-induced AKT but not ERK1/2 dephosphorylation. SK-MEL-28 and SK-MEL-5 cells were treated with PG at a concentration of $\mathrm{IC}_{50}$ for $15 \mathrm{~min}$ to $1 \mathrm{~h}$. Phosphorylation levels were visualized by Immunoblotting. Actin was used as a loading control. 
A

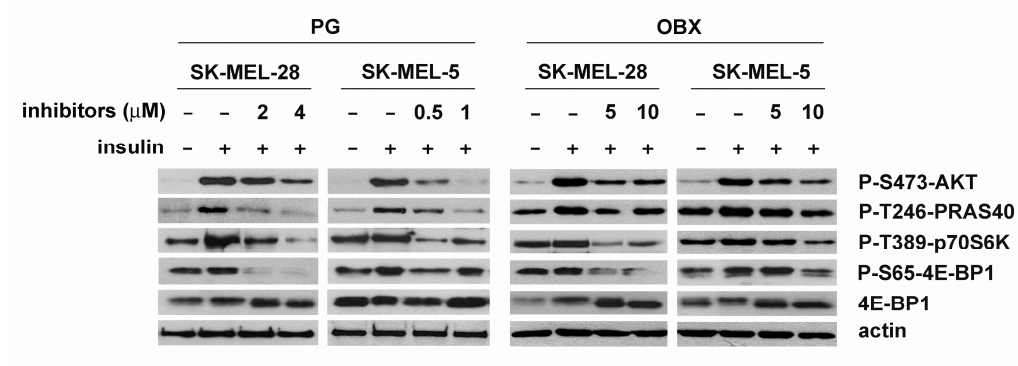

B

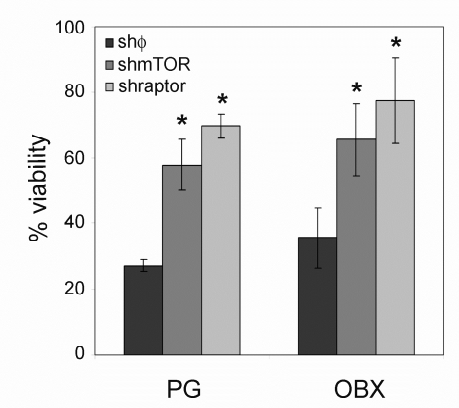

Fig. 3. Prodiginines target mTOR pathway. (A) Effect of prodiginines on critical effectors of PI3K/AKT/mTOR pathway in melanoma cells. SK-MEL-28 and SK-MEL-5 cells were deprived for $24 \mathrm{~h}$ and treated with PG or OBX at the indicated concentrations for 1 $\mathrm{h}$ before insulin-stimulation at $1 \mu \mathrm{M}$ for $30 \mathrm{~min}$. Cell extracts were analyzed by Immunoblotting. Actin was used as loading control. (B) Inhibition of mTOR activity prevents from prodiginines-mediated cell death. Stable control and knockdown cells were treated with PG or OBX at $4 \mu \mathrm{M}$ for $48 \mathrm{~h}$. The percentage of viable cells was calculated as the ratio of $\mathrm{A}_{570}$ between treated and control cells. Values are shown as mean \pm S.E.M. of three independent experiments performed in triplicate. Statistical significance is shown as *, $\mathrm{P}<0.01$. 
A
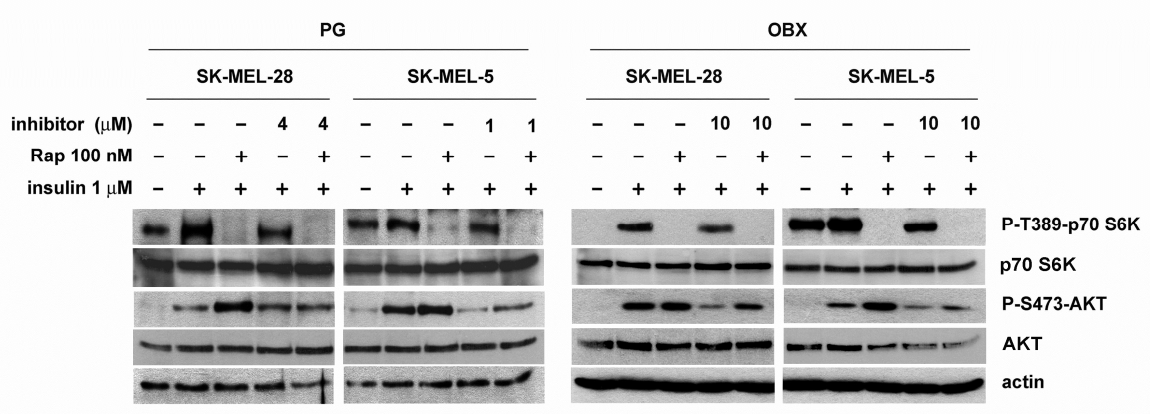
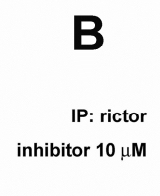

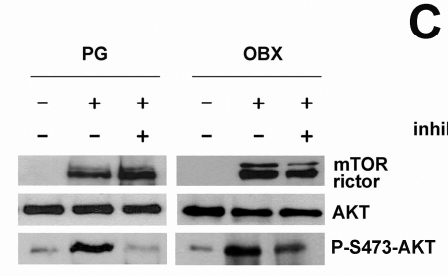

C
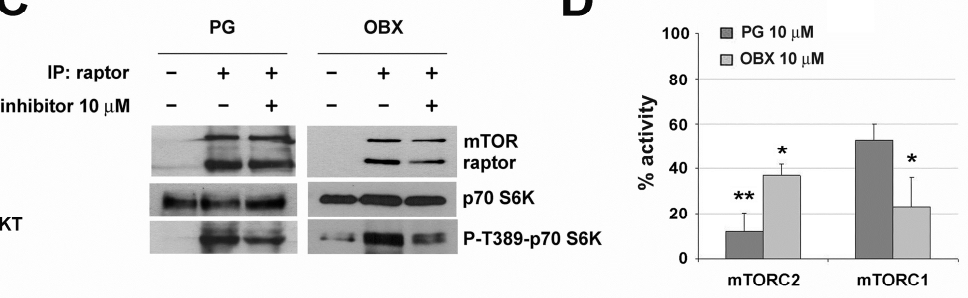

Fig. 4. Prodiginines counteract S6K1/IGF-1R negative feedback loop through mTORC1 and mTORC2 inhibition. (A) Effect of PG on S6K1/IGF-1R negative feedback loop in melanoma cells. Before insulin stimulation, deprived cells were pre-incubated with rapamycin $100 \mathrm{nM}$ for $15 \mathrm{~min}$ and prodiginines were then added at the indicated concentrations for $1 \mathrm{~h}$. (B) Prodiginines inhibit mTORC2 and mTORC1 complexes. Active mTORC2 and mTORC1 complexes were immunoprecipitated from insulin-stimulated SK-MEL-5 cells using anti-rictor or (C) anti-raptor antibodies, respectively. Immunoprecipitated were incubated with recombinant AKT or p70S6K (20 ng), $50 \mathrm{mM}$ Tris $\mathrm{pH} 7.5,10 \mathrm{mM}$ magnesium chloride, $10 \mu \mathrm{M}$ ATP and PG or OBX, both at $10 \mu \mathrm{M}$ for $30 \mathrm{~min}$. Kinase reaction was stopped with Laemmli buffer $(5 \mathrm{X})$ and phosphorylation levels of p70 S6K or AKT were visualized by Immunoblotting. (D) Data is expressed as the percentage of phospho-AKT or phospho-p70S6K levels (normalized by AKT or p70S6K levels), respectively, and shown as the mean \pm S.D. Statistical significance is shown as *, $\mathrm{P}<0.05$; **, $\mathrm{P}<0.01$. 


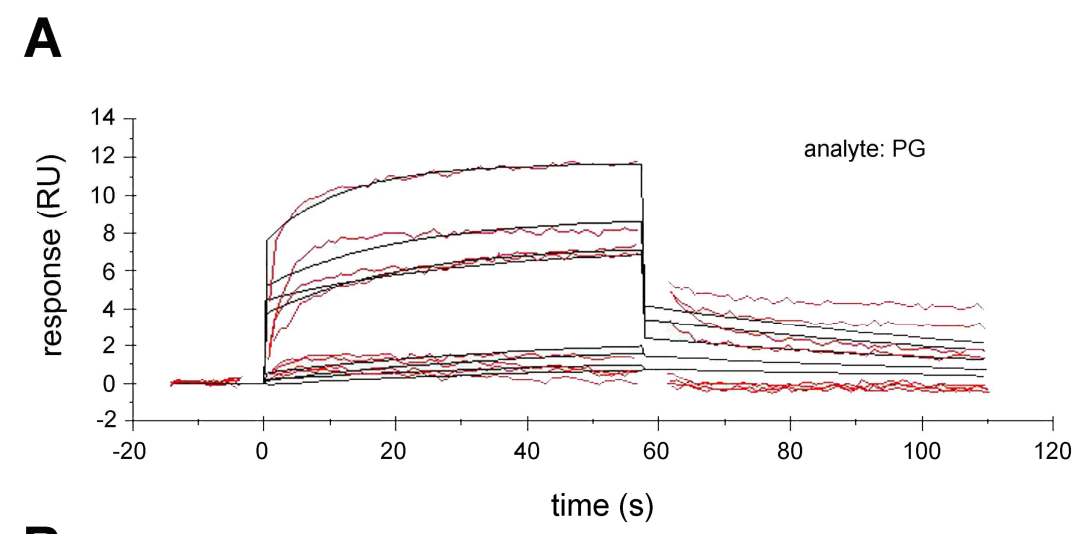

B

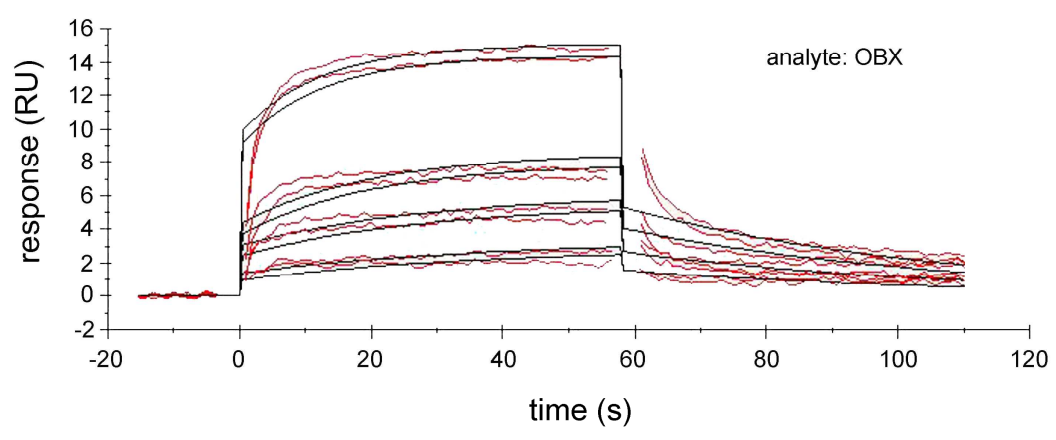

Fig. 5. Kinetic characterization of $P G$ and $O B X$ binding to mTOR by Surface Plasmon

Resonance (SPR) assays. (A) PG or (B) OBX were injected at a range of concentrations between $0.06 \mu \mathrm{M}$ to $1 \mu \mathrm{M}$ over immobilized mTOR (aa 1360-2549). Sensograms and curve fit (black lines) are shown. Kinetics and affinity evaluation of these sensograms showed affinity $\left(\mathrm{K}_{\mathrm{D}}=\mathrm{Kd} / \mathrm{Ka}\right)$ constants of $416 \pm 49 \mathrm{nM}$ for $\mathrm{PG}$ and $355 \pm 121 \mathrm{nM}$ for OBX, respectively. RU, resonance units. 


\begin{tabular}{|c|c|c|c|c|c|c|c|}
\multirow{2}{*}{ A } & \multicolumn{2}{|c|}{ PDK-1 } & \multicolumn{2}{c|}{ PKC-alpha } & \multicolumn{3}{c|}{ mTOR } \\
\cline { 2 - 8 } & LAA & PG & LW4 & PG & pp242 & PG & OBX \\
\hline $\begin{array}{c}\text { Initial } \\
\text { Score }\end{array}$ & -9.2 & -6.2 & -12.0 & -7.1 & -7.9 & -7.4 & -8.5 \\
\hline $\begin{array}{c}\text { Induced } \\
\text { Score }\end{array}$ & -10.1 & -6.6 & -11.5 & -7.6 & -9.7 & -10.1 & -10.3 \\
\hline $\begin{array}{c}\text { Induced } \\
\text { RMSD }\end{array}$ & 1.1 & 1.77 & 1.3 & 1.4 & 1.7 & 2.1 & 2.3 \\
\hline
\end{tabular}
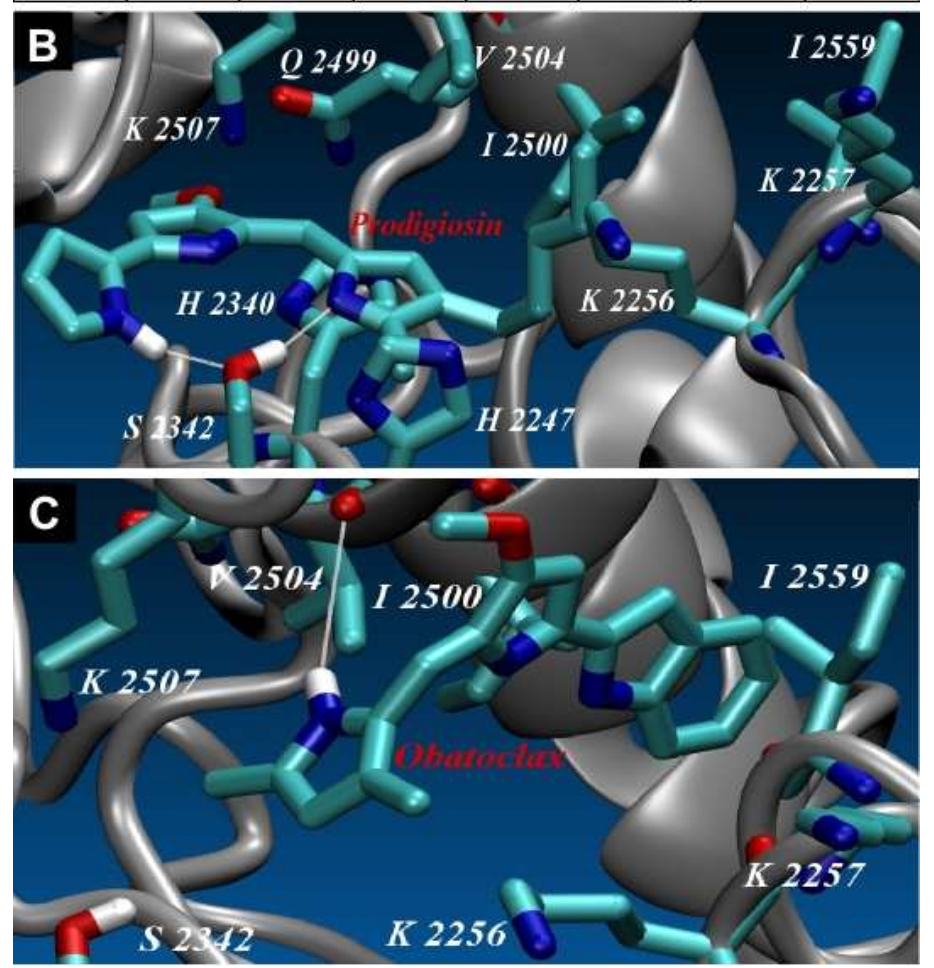

Fig. 6. In silico model. (A) Initial and after PELE (induced fit) scores obtained with Glide for the different systems and ligands. Also show is the RMSD along the induced fit process. RMSD was computed as all heavy atom within $10 \AA$ of the initial docking position (units in angstroms). (B) Detailed view of the PG-mTOR and (C) OBX-mTOR induced fit complex. 